\title{
Application of IoT and Machine Learning in Agriculture
}

\author{
Aman Kumar Dewangan \\ Department of Electrical Engineering \\ National Institute of Technology Raipur \\ Raipur, Chhattisgarh, India
}

\begin{abstract}
Agriculture is one of the major sources of economy in the country. Precision Agriculture is already in implementation in other countries but there is a need to implement, improve and evolve IoT(Internet of Things) and cloud computing technologies for better production of the crop. There is a steady increase in demand with population growth. Modernization in agriculture reduces dependency on individual human labor and land. The technology allows operational devising and accelerates verdict making on Farms. IoT allows us to accumulate surrounding data, stock it, concoct it and disseminate the information. The adoption of cloud computing has undergone a huge rise in need and would continue to grow in the coming future with improved cloud hosting and processing dexterities. AI(Artificial Intelligence) and IoT is a great lead as a solution to increased productivity. The data through IoT devices is made available publically for research purposes as data sets and is processed and examined for further prediction related to the crop being produced. The Traditional Technique of farming does not involve any process such as seed selection, soil analysis, weather analysis, vegetation analysis, Nutrient analysis if all these factors are taken in care, this all would bring a drastic change in the society. The System also has a block-chain based shipping system to ensure proper distribution without any wastage. Though the pen-paper tradition is hard to be replaced but minimizes a lot of work, moreover the analysis of manual work required can also be analyzed. A more intricate approach to IoT products in agriculture can be represented by the so-called farm productivity management systems. They usually include several agriculture IoT devices and sensors, installed on the premises as well as a powerful dashboard with analytical capabilities and inbuilt accounting/reporting features. In addition to the listed IoT agriculture use cases, some prominent opportunities include vehicle tracking (or even automation), storage management, logistics, etc.
\end{abstract}

Keywords-Internet of Things(IoT), Artificial Intelligence(AI), Machine Learning(ML), Smart Agriculture

\section{INTRODUCTION}

With the population boom, there is a rapid increase in the demand for food and agricultural stocks and cultivation process to improve yield, cost-effectiveness, and quality of crops/agricultural products being produced with new technology such as the Internet of Things(IoT) and Artificial Intelligence. There is a need to increase yield, effectiveness and enhanced production of land per unit area taken under deliberation. It is necessary to embrace new technologies to overcome these problems. There are various benefits associated with the enactment of new technologies which include: increased productivity, proper crop distribution, Crop pattern suggestion, proper utilization of resources such as
Fertilizers and manures using the technique of Automation and AI model.

\section{OBJECTIVE}

The Motive of paper is to detect and determine the nature and quality of soil based in a particular area, considering the toxicity level at present instance of time and predict it's future value using AI model. The Main objectives of the paper are:-

i. We have a limited amount of resources and their proper utilization is a subject of great concern, whether it's the utilization of water or utilization of minerals from ores all this indirectly affects our lives.

ii. Moreover, there is a lack of research data in this field, this would produce a huge collection of data for the farmers.Data, tons of data, collected by smart agriculture sensors, e.g. weather conditions, soil quality, crop's growth progress or cattle's health. This data can be used to track the state of your business in general as well as staff performance, equipment efficiency, etc.

iii. Better control over the internal processes and, as a result, lower production risks. The ability to foresee the output of your production allows you to plan for better product distribution.

iv. Being able to see any anomalies in the crop growth or livestock health, you will be able to mitigate the risks of losing your yield.

v. Increased business efficiency through process automation. By using smart devices, you can automate multiple processes across your production cycle, e.g. irrigation, fertilizing, or pest control.

Enhanced product quality and volumes. Achieve better control over the production process and maintain higher standards of crop quality and growth capacity through automation.

\section{LITERATURE SURVEY}

In the modern synopsis of the industrial revolution, 4.0 where we have a limited amount of resources and their proper utilization is a subject of great concern, whether it's the utilization of water or utilization of minerals from ores all this indirectly affects our lives. With the limited availability of resources and increased consumption there prices have been 
rising up and so there sustainable utilization is necessary. Similarly, in the case of Farming where we need to feed a large number of customers, any kind of loss at any stage proves to be a huge loss to the economy and the user as well. Moreover, there is a lack of research data in this field.The main motive is to bring IoT and Machine Applied Farming to India, to ample up the technical application of AI and Machine Learning among Farmers, Researchers, and Government.

\section{PROTOTYPE MODULE}

The scheme structural design illustration in Fig. represents the whole architecture of this outline. It points information stream throughout the System. The sensor values used for the same cover temperature and wetness of the atmosphere, the moisture content in the soil, it's $\mathrm{pH}$ state, salinity, NO2 concentration, and a Unified Water System. The data are received by Google Firebase where the database resides and the Web Server is hosted on Apache Web Server.

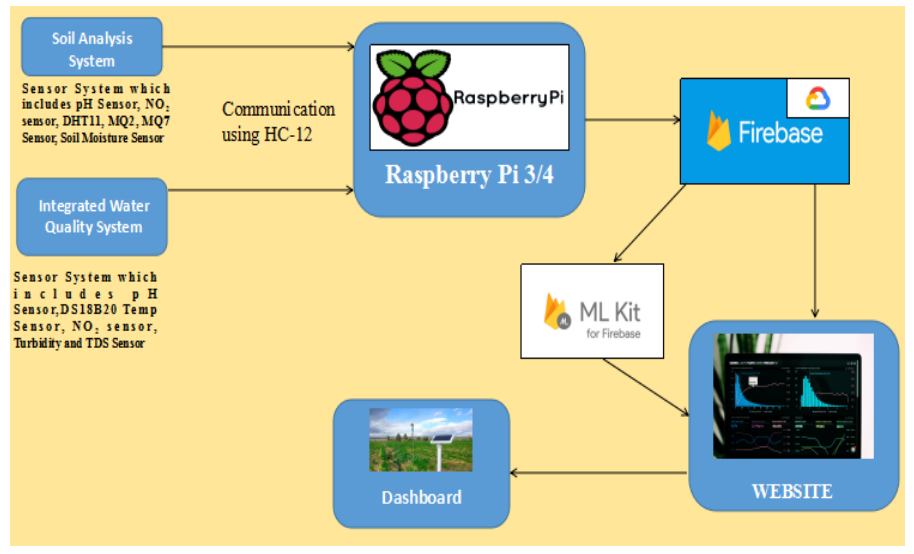

V. TECHNICAL REQUIREMENTS

\section{A. Hardware Requirement}

Arduino Mega2560 - The Arduino Mega 2560 is a microcontroller board based on the ATmega2560. It has 54 digital input/output pins (of which 15 can be used as PWM outputs), 16 analog inputs, 4 UARTs (hardware serial gates), a $16 \mathrm{MHz}$ crystal oscillator, a USB attachment, a power tool, an ICSP header, and a reset key. It comprises

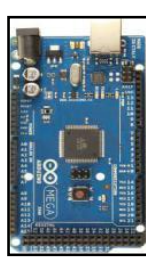
everything required to assist the microcontroller; simply connect it to a computer with a USB cable or power it with an AC-to-DC adapter or battery to use.

- Temperature and humidity sensor - DHT22 is a lowcost digital sensor that uses a thermistor to measure the air in the surrounding and it uses a capacitive humidity sensor to measure humidity. They consist of an NTC temperature sensor/Thermistor to measure temperature. A thermistor is a thermal resistor - a resistor that adjusts its resistance with temperature. Technically, all resistors are thermistors their resistance varies slightly with temperature - but the change is usually very very small and difficult to measure. The humidity sensing element is used, of course, to measure humidity, which has two electrodes with moisture-holding a substrate (usually a pinch of salt or conductive plastic polymer) sandwiched within them. The variation in resistance between the two electrodes is equivalent to the relative humidity. The ratio of moisture in the air to the highest amount of moisture at a particular air temperature is called relative humidity.

The typical input voltage range is 3 to $5 \mathrm{~V}$ and the maximum current allowable is $2.5 \mathrm{~mA}$. It is good for $-40^{\circ} \mathrm{C}$ to $15^{\circ} \mathrm{C}$ temperature readings with +0.5 or $-0.5^{\circ} \mathrm{C}$ accuracy and $0-100 \%$ humidity readings with $2-5 \%$ accuracy.

- DS18B20 Temperature Sensor - The digital temperature sensor DS18B20 follows a single wire protocol and it can be used to estimate the temperature in the range of $-67^{\circ} \mathrm{F}$ to $+257^{\circ} \mathrm{F}$ or $55^{\circ} \mathrm{C}$ to $+125^{\circ} \mathrm{C}$ with $+-5 \%$ accuracy. The spectrum of acquired data from the 1-wire can range from 9-bit to 12-bit. Because, this sensor supports the single wire protocol, and the regulating of this can be done through an only pin of Microcontroller. This is a superior level

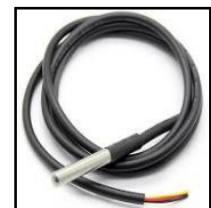
protocol, where each sensor can be set with a 64-bit serial code which serves to control copious sensors using a single pin of the microcontroller.It is a part of the integrated Water System.Use a zero before decimal points: “0.25," not “.25." Use "cm3," not "cc.” (bullet list)

- MiCS-4514 $\mathrm{NO}_{2}$ Sensor - The MiCS-4514 is a compressed MOS Sensor with two fully autonomous sensing elements of one unit. Sensitivity Factor is determined as $\mathrm{R}_{\mathrm{s}}$ in air divided by $\mathrm{R}_{\mathrm{s}}$ at 60 ppm CO. Test conditions are $23^{\circ} \mathrm{C}$ with an error of $5^{\circ} \mathrm{C}$ and $50 \mathrm{R}_{\mathrm{h}}$ with an error of $10 \%$.Sensitivity Factor is defined as $R_{S}$ at $0.25 \mathrm{ppm} \mathrm{NO} \mathrm{N}_{2}$ divided by $\mathrm{R}_{\mathrm{s}}$ in air. Test conditions are $23^{\circ} \mathrm{C}$ with an error of $5^{\circ} \mathrm{C}$ and $50 \mathrm{R}_{\mathrm{h}}$ with an error of less than of equal to $5 \%$.The Silicon Gas sensor structure consists of an explicitly micromachined diaphragm with an enclosed heating resistor and sensor panel on top. The MiCS-4514

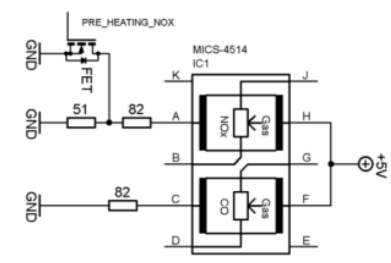
incorporates two sensor chips with independent heaters and sensitive layers. One sensor chip detects oxidizing gases $(\mathrm{OX})$ and another sensor detects reducing gases(RED).

- $\underline{\mathrm{HC}-12}$ - HC-12 radio serial port communication module is a newgeneration multichannel implanted broadcast data transmission module. Its radio operating frequency band is 
433.4-473.0MHz, multiple channels can be set, with the stepping of $400 \mathrm{kHz}$, and there are a total of 100 channels. The maximum transmitting capability of the module is $100 \mathrm{~mW}(20 \mathrm{dBm})$, the receiving sensitivity is $-117 \mathrm{dBm}$ at a baud flow of $5,000 \mathrm{bps}$ in the air, and the transmission distance is $1,000 \mathrm{~m}$ in open space. There is MCU inside the module, and the user doesn't need to program the module separately, and all transparent transmission mode is only reliable for receiving and sending serial port data, so it is convenient to use. The module utilizes multiple serial port transparent synchromesh modes, and the user could select them by AT command according to user requirements. The average working current of three modes FU1, FU2 and FU3 in idle state is $80 \mu \mathrm{a}$, $3.6 \mathrm{~mA}$, and $16 \mathrm{~mA}$ respectively, and the maximum working current is $100 \mathrm{~mA}$ (in transmitting state).

- Raspberry Pi - The Raspberry Pi is a low cost, credit card-sized microcomputer that plugs into a computer monitor or TV, and uses a standard keyboard and mouse. It also has very low power dissipation between $0.5 \mathrm{~W}$ and $1 \mathrm{~W}$. New Out Of Box Software (NOOBS) gives the user a choice of the operating system from the standard distributions. Raspbian is the approved operating system for

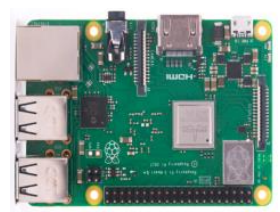
normal use on a Raspberry Pi. In this project, Raspberry $\mathrm{Pi}$ essentially demands two purposes. First, server and second for storage. Apache Web Server is hosted on this Pi along with a Firebase database for the accommodation of the data.

- $\mathrm{pH}$ Sensor - The $\mathrm{pH}$ sensor module consists of a $\mathrm{pH}$ sensor also called a pH probe and a signal conditioning board which provides an output that is proportionate to the $\mathrm{pH}$ value and can be interfaced straight with any microcontroller. The $\mathrm{pH}$ sensor has an OxidationReduction Potential(ORP) Probe which reflects the voltage

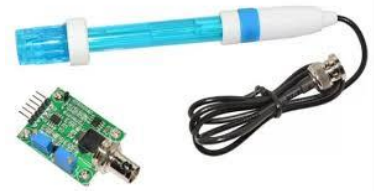
proportional to the tendency of the clarification to gain or lose electrons from other substances. This voltage and $\mathrm{pH}$ have a reducing dependence, lower the voltage higher the $\mathrm{pH}$ obtained.

- MQ2 Sensor - Sensitive material of the MQ-2 gas sensor is $\mathrm{SnO} 2$, which with lower conductivity in clean air. When the target ignitable gas exists, The sensor's conductivity is higher along with the gas concentration rising. It converts the variation of conductivity to compare the output signal of gas concentration. MQ-2 gas sensor has a high sensibility to LPG, Propane, and Hydrogen, also could be used to Methane and other combustible steam, it is with low cost and suitable for different application. It can estimate the concentration of ignitable gases up to a range of $300-10000 \mathrm{ppm}$. It has a conventional encapsulation of Bakelite.

- Turbidity Sensor - The turbidity sensor detects water quality by estimating the levels of turbidity. It adopts light to detect suspended particles in water by measuring the light transmittance and scattering rate, which varies with the amount of total suspended solids (TSS) in water. As the TTS increases, the liquid turbidity level increases. Turbidity sensors are used to

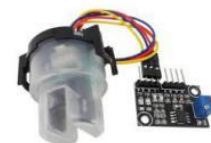
regulate water quality in rivers and streams, wastewater and effluent determinations, control instrumentation for settling ponds, sediment transport research and laboratory measurements. This sensor provides analog and digital signal output modes. The threshold is adaptable when in digital signal mode. You can select the mode according to your MCU. If you leave the sensor in the pure water, that is NTU < 0.5 , it should output " $4.1 \pm 0.3 \mathrm{~V}$ " when the temperature is $10^{\circ} \sim 50^{\circ} \mathrm{C}$. The Operating Temperature of Turbidity meter is $5^{\circ} \sim 90^{\circ} \mathrm{C}$.

- Total Dissolved Solid(TDS) Sensor - TDS (Total Dissolved Solids) indicates how many milligrams of soluble solids dissolved in one liter of water. In general, the higher the TDS value, the more soluble solids dissolved in water, and the less clean the water is. Therefore, the TDS value can be used as one of the references for reflecting the cleanliness of the water. TDS pen is widely used equipment to measure TDS value. The excitation source is an AC signal, which can effectively prevent the probe

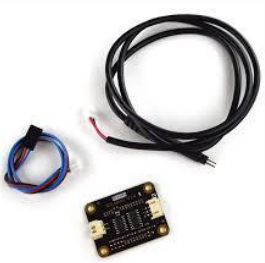
from polarization and prolong the life of the probe, meanwhile, increase the stability of the output signal. The TDS probe is waterproof, it can be submerged in water for long time determination. The probe can not be used in water above 55 degrees centigrade.TDS Measurement Range: $0 \sim 1000$ ppm TDS Measurement Accuracy: $\pm 10 \%$ F.S. $\left(25^{\circ} \mathrm{C}\right)$

\section{B. Software Requirement}

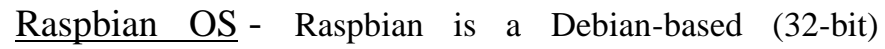
computer operating system for Raspberry Pi. There are numerous variants of Raspbian including Raspbian Stretch and Raspbian Jessie. Though Raspbian is not designed to work the Pi like a desktop computer provides the users with an LXDE desktop background. The Pi ingests not have a great deal of processor speed or memory, but it

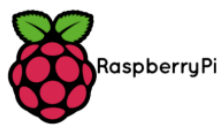
does have sufficient support to run LXDE and some of the applications like the simplistic Epiphany web browser and several more further. 
Arduino Software - The Arduino Integrated Development Environment (IDE) is a cross-platform application (for Windows, macOS, Linux) that is written in functions from $\mathrm{C}$ and $\mathrm{C}++$. Arduino is an open-source electronics platform based on easy-to-use hardware and software. It is used to write and upload programs to Arduino compatible boards, but also, with the help of 3rd party centers, other vendor development boards. The Arduino IDE supports the languages $\mathrm{C}$ and $\mathrm{C}++$ using special rules of

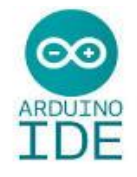
code structuring. The Arduino IDE employs the program avrdude to convert the executable code into a text file in the hexadecimal encoding that is loaded into the Arduino board by a loader program in the board's firmware. By getting inputs from sensors, Arduino senses the environment and affects or influences its surroundings with different actions and actuators. Arduino is obliged to compile and upload the necessary codes to run the UNO.

Apache Web Server - Apache web server is a free and open-source cross-platform web server software developed and maintained by Apache Software Foundation. It powers around $40 \%$ of websites around the world. It is quick, stable, and safe. It can be profoundly customized to satisfy the demands of many different environments by using branches and modules. In this project, this server is needed to monitor Apache Web Server the received data and perform the
necessary assignments after the data representation and processing. The server is what is responsible for monitoring the health of the crops and also to take control of the automated water sprinkling system. In this project, the server is hosted on the Raspberry Pi.

Firebase - The Firebase Realtime Database is cloudhosted. Data is stored as JSON and synchronized in real-time to every connected client. When you develop cross-platform apps with our iOS, Android, and JavaScript SDKs, all of your clients share one Realtime Database occurrence and automatically

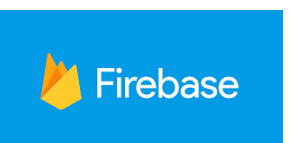
collect updates with the most current data. Firebase Realtime Database allows you to develop rich, collaborative applications by enabling secure access to the database directly from client-side code. Data is persevered locally, and even while offline, real-time events continue to fire, giving the end-user a responsive experience. When the device retrieves connection, the Realtime Database synchronizes the local data changes with the remote updates that occurred while the client was offline, merging any conflicts automatically
VI. SCHEME MODULE
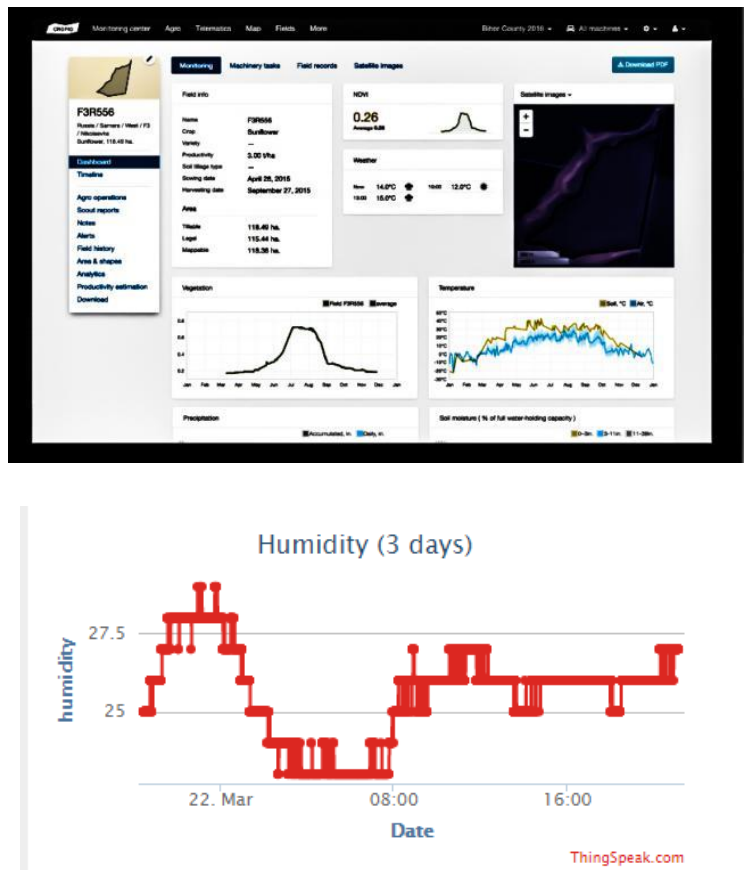

Dust Concentration (3 days)

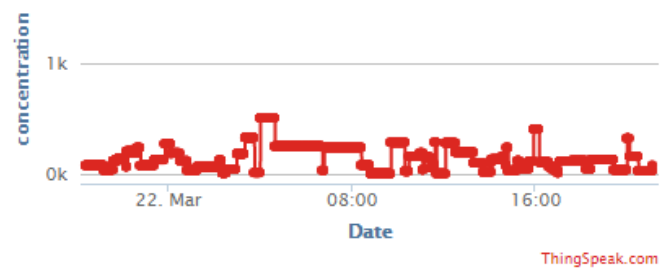

Fig. .....Dashboard Visualization, Dust Concentration and Humidity.

The offered practice is implementing the notion of IoT in the farming field by using smart sensors to make agriculture field a smarter one. The primary purpose of the project is to accumulate data of multiple nodes and to process this information. The producers will be capable to regulate the actions remotely through a mobile application as well as access the accounts through a cloud. The design of the scheme is to generate centralize monitoring and control for the agricultural land. This can be maintained and operated from any place wirelessly using a mobile device. The application user can control fundamental services of accumulation of environmental, soil, fertilization, and irrigation data; automatically associate such data and filter out illogical data from the prospect of appraising crop performance; and figure crop forecasts and personalized product suggestions for any selective farm using the application. The system can integrate virtually any IoT device, including commercially accessible sensors, cameras, weather stations, etc. and can form a bunch of these materials which would execute it adaptable for a single user to cover a large region and reserve their data in the cloud for 
performance interpretation and suggestions. End-user can get all these aspects of the field on Smartphone by an administration Smart Agro Services and can guide the operations. The sensor network is devised to acquire information concerning the climatic requirements of the farm such as Soil Moisture, Temperature, Light, and Humidity. With the help of this, the scheme will determine the operations on the field. A single farm can have various crops divided into fields. So a particular crop will have several parameters to be constrained. This, we need to have a batch that will collect data separately. These nodes are installed on various parts on the field depending upon the parameters. Each The node comprises of a microprocessor Raspberry Pi and a sensor connected to it. Sensors may be temperature and humidity sensor or soil moisture sensors. The soil moisture sensor being an analog sensor requires an ADC (Analog to Digital Converter). The data from the sensor is in the analog form and needs to be converted to digital form. Hence the raw data is supplied to ADC which in turn to digital. The digital data is in the form of voltage value and depending on voltage value the percentage of moisture in the soil is taken. The sensors are connected to Raspberry Pi. Raspberry Pi collects the data from the sensors of that node. There are multiple nodes that are placed around the field. Using the clustering technology the farmer can make accurate decisions like in which part of the field soil moisture has reduced and where to divert the irrigation system.As well as when to switch on and off the motor pumps and other devices for the parameters to be maintained. Data from all these nodes are collected and transferred to a cloud. Here, we are using the cloud service as a storage database. The Data sent to the cloud is stored in the cloud database. Farmers can log in to their respective accounts to view their history and the current data of each node. The data from the cloud is given to the mobile application. With the help of the mobile application, the farmers get ease to control various devices and record the readings from the sensors.

\section{SMART MOBILE APPLICATION:}

The planters are the end-users of this application. This application will provide full radio connectivity to the farmers for their farm. The concocted data from the cloud will be obtained by the farmers using the mobile application. The farmers will get a graphical illustration of data as well for more suitable comprehension of the parameters. With the help of this data summary, the farmers will be aware of the climatic circumstances of the farm and consequently will constrain the accessories such as light and motor pumps. Each farmer will have its own account by which he can log in utilizing an individual username and password. New users can record using their email ID and create a new account. The farmers will get the direct readings from the sensors for the node they have selected. Based on this they will also get an alert for which device should be switched on or off. The operators can thus check for the readings and wirelessly regulate the devices for the field. Apart from this the application also provides other beneficial services for the farmers as follows:

\section{CONCLUSION}

This Paper proposed an IoT and AI-based scheme for the Agricultural Sector consisting of an assemblage of Microcontrollers, sensors, and a Consolidated Water Quality System. In this method, the data consolidated from Sensors will be transferred to Cloud for processing and Data set organization through the Internet. The real-time data will be fed into an ML algorithm after a certain fixed interval of time, based on which it would predict the soil condition of the system. For predicting the variable parameter of Soil, the Regression method would be used but checking the Toxicity of Water Anomaly detection method is most suitable.

This system will be aid provided to the farmers for digitalizing agriculture. Approximating possible work on this system may entail accumulating the data and rendering services for each crop exclusively.

\section{REFERENCES}

[1] S.R. Juhi Reshma, Anitha S. Pillai from book Proceedings of the Eighth International Conference on Soft Computing and Pattern Recognition (pp.602-613)

[2] Abhishek L, Rishi Barath B International Journal of Innovative Technology and Exploring Engineering (IJITEE) ISSN: 2278-3075, Volume-8 Issue-8 June, 2019.

[3] SanatSarangia, JayalakshmiUmadikar, Subrat Kara, "Automation of Agriculture Support Systems using Wisekar: Case study of a crop disease advisory service", 2016

[4] MUHAMMAD AYAZ, MOHAMMAD AMMAD-UDDIN, ZUBAIR SHARIF, ALI MANSOUR, AND EL-HADI M. AGGOUNE Senso Networks and Cellular Systems Research Center, University of Tabuk, Tabuk 71491, Saudi Arabia 2CS Department, COMSATS University Islamabad, Sahiwal 57000, Pakistan 3Lab-STICC, UMR 6285 CNRS ENSTA Bretagne, 29806 Brest, France(IEEE Access).

[5] Fabrizio Balducci, Donato Impedovo and Giuseppe Pirlo Dipartimento di Informatica, Università degli studi di Bari Aldo Moro, 70125 Bari, Italy.

[6] Agraj Aher, Janhavi Kasar, Palasha Ahuja, Varsha Jadhav Internationa Research Journal of Engineering and Technology (IRJET) Volume: 05 Issue: 03 | Mar-2018

[7] Prof. K. A. Patil, Prof. N. R. Kale “A Model for Smart Agriculture Using IoT" IEEE $\mid$ December 2016. 